\title{
Research on Partial Discharge Detection methods for Electrical Equipment Diagnosis
}

\author{
Xie Xinnan ${ }^{1}$, Mu Jinglong ${ }^{1}$, Liu Ren ${ }^{1}$, Meng Qingguo ${ }^{2}$, Wu Shaoyong ${ }^{1}$, Lv Pin ${ }^{2}$, Sun \\ Fengwei ${ }^{1}$, Liu Bo ${ }^{1}$, Zhang Bo ${ }^{1}$, Zhu Bo ${ }^{1}$, Zhang Fuliang ${ }^{1}$, Liu Peng ${ }^{1}$ \\ ${ }^{1}$ Fushun Power Supply Company, Liaoning Electric Power Company Limited, State Grid, China \\ ${ }^{2}$ Manzhouli City Power Supply Company, East Inner Mongolia Electric Power Company Limited, \\ State Grid, China
}

fushunpowersupply@163.com

Keywords: Partial discharged detection, Ultrasonic wave Low voltage transient, Troubleshooting

\begin{abstract}
Switchgear charged for fault detection, principles and methods the live test from switchgear partial discharge, analyzes the use of ultrasonic detection assays and transient voltage for the switchgear cabinet feasibility of fault diagnosis; using a combination of switchgear partial discharge founding live testing equipment failure cases, by power testing experimental analysis confirmed the presence of switchgear failure to verify the validity of partial discharge detection in live test switchgear fault diagnosis.
\end{abstract}

\section{Introduction}

$10 \mathrm{kV}$ and $35 \mathrm{kV}$ voltage level in the system, there is such a wide range applications of high voltage switchgear. Switchgear bear fed directly to the user's task, however is difficult most of the feeder line as transmission line in accordance with the "N-1" principle reliability configuration, therefore the reliability of switchgear equipment directly determines the reliability and security of electricity supply safe. According to "Zhejiang transmission equipment state maintenance test procedures" requirement for good equipment of state evaluation, its maintenance cycle may be extended to 5.5 years. With the equipment maintenance cycle extend and condition-based maintenance the depth development, switchgear power test opportunity fewer, live detect and on-line monitoring in a certain extent determines the status of the switchgear judgment. This paper analyzes the case of equipment failure cases by ultrasound and transient ground voltage live test detection means discovered shows partial discharge live detection effectiveness and importance in the diagnosis of insulated switchgear[1-3].

\section{Partial discharge detection methods}

(1) Ultrasonic test method

Before the electrical equipment partial discharge, discharge points around the electric stress, media stress, particle force in a relatively balanced state. Partial discharge is a quick release or charge migration, causing an electric field stress; mechanical stress and particle force around the discharge point loss of equilibrium oscillate change process. Meanwhile, because of the rapid oscillations of the mechanical stress and force the particles, resulting in vibration phenomena around the medium of the discharge point, thereby generating the acoustic signal, acoustic wave spectrum is very wide, from a few tens $\mathrm{Hz}$ to several $\mathrm{MHz}$, the ultrasonic detection frequency bands above mainly $20 \mathrm{kHz}$ ultrasound, thereby avoiding disturbance [1]. Also, because the vibrations and the acoustic wave propagation process is loss, differences in transmission resulting in the complex proportional relationship between the ultrasonic intensity and discharge intensity, quantitative analysis is more difficult, but also severe signal attenuation less prone to outside interference. Therefore, the method is to use the ultrasonic testing, high accuracy of the test.

(2) Transient earth voltage test method

When the partial discharge occurs, the electronic migrate fast from charged body to ground 
non-charged body (such as cabinet), while the discharge point to generate high-frequency current wave propagation in both directions. Affected by the skin effect, the current wave is concentrated on the surface of the metal cabinet spread, while not directly penetrate. However, disconnected or insulated metal junction, the current wave can be transferred to the outer surface, and in the form of electromagnetic waves into free space, when the rising edge of electromagnetic waves encounter the outer surface of the metal, it creates to generate transient voltage to ground. Transient voltage available to TEV sensors measure, the amplitude is related to extent of attenuation discharge amount and route of transmission the amount of attenuation depends on the internal structure of the discharge point location, equipment, and size of the opening[4, 5]. Use time difference of arrival in the measured signal of two TEV sensors installed on the outer surface of the metal cabinet can be achieved roughly the partial discharge location.

\section{Cases of switchgear fault diagnosis}

(1) Charged detection process

A routine inspection is conducted to detect PD charged for a $35 \mathrm{kV}$ switchgear substation. The type of switchgear is KYN-40.5-0.4. The ambient air humidity is $43 \%$ and room temperature is $12^{\circ} \mathrm{C}$ during the test. Firstly, the team uses the switchgear partial discharge test instrument for ultrasonic testing, gain selection for 100 , background amplitude of the test for $-7 \mathrm{~dB}$, ultrasound test results are shown in Table 1. As can be seen from Table 1, \#2, the ultrasonic amplitude just above the $35 \mathrm{kV}$ main transformer switch cabinet bus bridge is $36 \mathrm{~dB}$, while the adjacent ultrasonic amplitude between two parts of the same interval less than $6 \mathrm{~dB}$. According to "Zhejiang Power Transmission Equipment Charged testing procedures", it is suspected that 2 \# main transformer $35 \mathrm{kV}$ switchgear bus bar has discharge defects.

Table 1 Ultrasonic testing data

\begin{tabular}{|c|c|c|}
\hline \multicolumn{3}{|c|}{ Ultrasound background: $-7 \mathrm{~dB}$} \\
\hline \multirow{2}{*}{ Cabinet name } & Ultrasonic measurements $(\mathrm{dB})$ \\
\cline { 2 - 3 } & Front of cabinet & Front of cabinet \\
\hline Jiahe 3525 & -4 & -4 \\
\hline 1\# main transformer 35kV breaker & -4 & -5 \\
\hline 35kV bus I voltage transformer & -3 & -4 \\
\hline Jianggang 3527 breaker & -4 & -4 \\
\hline Jianggang 3527 voltage & -4 & -4 \\
\hline transformer & -5 & -5 \\
\hline 35kV Bus I sub-switch & -6 & -6 \\
\hline 35kV Bus II sub-switch contactor & -3 & -5 \\
\hline Jiangdian 3528 breaker & -4 & 1 \\
\hline Jiangdian 3528 voltage & & 5 \\
\hline transformer & 36 & 2 \\
\hline 1\# main transformer 35kV breaker & -5 & \\
\hline 35kV bus II voltage transformer & &
\end{tabular}

Use switchgear PD tester TEV test mode test three cabinet which are Jiang Dian 3528, 2 \# main transformer and 35kV II segment Bus Voltage Transformer, the test results are shown in Table 2.

As can be seen from table 2, the amplitude of TEV measurement were not greater than $20 \mathrm{~dB}$, the relative value is far less than the rules of $15 \mathrm{~dB}$ compared with the background value, the PD pulse value is greater than 1000, can not be identified as partial discharge signal. But the amplitude of the signal detected by the ultrasonic signal has reached $36 \mathrm{~dB}$, discharge obviously, this switch cabinet bus bridge defects, power should be treated immediately. 
Table 2 TEV test data

\begin{tabular}{|c|cc|cc|}
\hline \multicolumn{4}{|c|}{ Ultrasound background: $10 \mathrm{~dB}$, pulse: 0} \\
\hline \multirow{2}{*}{ Cabinet name } & \multicolumn{3}{|c|}{ TEV measured values $(\mathrm{dB})$} \\
\cline { 2 - 5 } & \multicolumn{2}{|c|}{ Front of cabinet } & Front of cabinet \\
\hline $\begin{array}{c}\text { Jiangdian } 3528 \text { voltage } \\
\text { transformer }\end{array}$ & Amplitude: 12 & pulse: 1500 & Amplitude: 16 & pulse: 2000 \\
\hline 2\# main transformer 35kV breaker & Amplitude: 13 & pulse: 6800 & Amplitude: 12 & pulse: 500 \\
\hline 35kV bus II voltage transformer & Amplitude: 15 & pulse: 2500 & Amplitude: 15 & pulse: 1500 \\
\hline
\end{tabular}

(2) Blackout test

To verify the scene with the detection analysis, blackout for defect elimination treatment, test personnel at the scene on the line between $2 \#$ main transformer bushing and $35 \mathrm{kVII}$ bus bridge (with little mother of $2 \#$ main transformer $35 \mathrm{kV} \mathrm{CT}$ ) do the insulation resistance test, the test results are shown in table 3 . The test environment temperature is 20 degrees, humidity is $65 \%$.

Table 3 Insulation test data

\begin{tabular}{|c|c|c|c|}
\hline Phase & A & B & C \\
\hline Insulating resistance $(\mathrm{M} \Omega)$ & 50000 & 320 & 50000 \\
\hline
\end{tabular}

As can be seen from table 3 that $\mathrm{B}$ phase insulation resistance is obviously on the low side, the normal qualified is $\mathrm{A} / \mathrm{C}$ phase. We can see that the $\mathrm{B}$ bus is abnormal, it also results in line with the live test mother bridge at maximum amplitude of ultrasonic is consistent, which further confirmed the $2 \#$ main transformer $35 \mathrm{kV}$ bus bridge defects, and confirmed the validity of field live detection results.

In order to further clarify the specific properties of defects, open the $2 \#$ main transformer into the wire bridge cabinet board inspection, found that the segregation baffle mother bridge between $\mathrm{B}$ phase and $\mathrm{C}$ phase down, and leaning heat shrinkable sleeve into wire bridge in B phase, the point of contact heat shrinkable sleeve has charred melted, which caused the metal exposure.

\section{Conclusion}

TEV and ultrasonic method for founding switchgear partial discharge is effective. However, the two methods of different defect nature of the reaction sensitivity is different, in this case of corona discharge, since the TEV mode detection signal in $3-100 \mathrm{MHz}$, when relatively strong corona, the pulse emitted by it is not in TEV detection frequency range within which does not reflect the exceptions. Ultrasonic method is more suitable for detecting air dielectric discharge, thus more sensitive corona discharge. So that we can draw the sound - electric joint detection is the best means of detection switchgear partial discharge detection.

\section{References}

[1] Zhao Hong. The voltage quality of 10KV distribution network analysis. Rural electrician, 2003

[2] Zhang Zhi-bao. Transformer principles and applications. Chemical Industry Press, 2007

[3] Fang Da-qian. Transformer quick checking and calculating Handbook. China water power Press, 2004

[4] Yin Ke-ning. Transformer design principle. China electric power press, 2003

[5] Zhonghong-bi, Gaozhan-bang. Power transformer maintenance and testing manual. China Electric Force Press, 1999. 